\title{
Systemic treatment with pulsed electromagnetic fields do not affect bone microarchitecture in osteoporotic rats
}

\author{
Olav P. van der Jagt • Jacqueline C. van der Linden • \\ Jan H. Waarsing • Jan A. N. Verhaar • Harrie Weinans
}

Received: 25 November 2011 / Accepted: 19 December 2011 / Published online: 17 January 2012

(C) The Author(s) 2012. This article is published with open access at Springerlink.com

\begin{abstract}
Purpose Pulsed electromagnetic fields (PEMF) are currently used in the treatment of spinal fusions and non-unions. There are indications that PEMF might also be effective in the treatment of osteoporosis. In this study we examined whether whole-body PEMF treatment affects the bone microarchitecture in an osteoporotic rat model.

Methods Twenty-week-old female rats were ovariectomised $(n=20)$. Four different PEMF treatment protocols based on previous experimental studies and based on clinically used PEMF signals were examined ( $2 \mathrm{~h} /$ day, 5 days/week). A control group did not receive PEMF. At zero, three and six weeks cancellous and cortical bone architectural changes at the proximal tibia were evaluated using in vivo microCT scanning. Results PEMF treatment did not induce any changes in cancellous or cortical bone compared to untreated controls. Conclusions Although previous studies have shown strong effects of PEMF in osteoporosis we were unable to demonstrate this in any of the treatment protocols. Using in vivo microCT scanning we were able to identify small bone changes in time. Subtle differences in the experimental setup might explain the differences in study outcomes in the literature. Since PEMF treatment is safe, future experimental studies on the effect of PEMF on bone can better be performed directly on humans, eliminating the potential translation issues between animals and humans. In this study we found no support for the use of PEMF in the treatment of osteoporosis.
\end{abstract}

O. P. van der Jagt $(\triangle) \cdot$ J. C. van der Linden $\cdot$ J. H. Waarsing

J. A. N. Verhaar $\cdot$ H. Weinans

Department of Orthopaedics, Erasmus MC,

University Medical Center Rotterdam,

Dr Molenwaterplein 40, PO Box 2040, 3000 CA

Rotterdam, The Netherlands

e-mail: o.vanderjagt@erasmusmc.nl

\section{Introduction}

Osteoporosis is a disease characterised by progressive bone loss and deterioration of the microarchitecture leading to an increased fracture risk. Osteoporosis can have distinctive causes including lack of sex hormones, long-term use of glucocorticoids or disuse. Current standard therapy consists of reducing further bone loss using bisphosphonates [1]. The use of bisphosphonates is accompanied by potential side effects such as gastrointestinal complaints, osteonecrosis of the jaw and atypical femoral fractures [2]. As an alternative treatment biophysical stimuli have long been proposed. These might be cheaper and induce fewer side effects. Pulsed electromagnetic fields (PEMF) might be one such treatment and indeed there is some evidence that these positively influence bone mass.

The finding that electrical currents are induced during mechanical loading of bone has led to the development of PEMF $[3,4]$. Much research has been done on the effects of PEMF on bone. In vitro studies do show that a variety of growth factors that are important in bone metabolism are affected, including bone morphogenetic protein 2 (BMP-2), transforming growth factor beta (TGF- $\beta$ ) and insulin-like growth factor II (IGF-II) [5-11]. Furthermore, PEMF result in the activation of extracellular signal-regulated kinase (ERK), mitogen-activated protein kinase (MAPK) and prostaglandin synthesis, which might also lead to stimulatory effects on bone [12-14]. Clinically PEMF are widely used for the treatment of non-unions, although they have never been proven to be effective in a prospective randomised controlled trial [15-22].

PEMF as treatment for osteoporosis have been studied before. In a clinical study it was shown that PEMF treatment of the wrist induced an increase in bone mineral density (BMD) in the distal radius of osteoporosis-prone women 
[23]. Re-examination 12 years later did show that these effects had lapsed [24]. Only a few animal studies have been published on the subject. In one study complete preventive effects of PEMF on trabecular bone loss in an ovariectomised rat model were demonstrated [12]. PEMF consisted of a single pulse wave form with a maximum of $8 \mathrm{G}$ and were applied eight hours per day. In another study it was shown that PEMF led to trabecular thickening in ovariectomised rats when treated with pulse bursts with a maximum of $9.6 \mathrm{G}$ for six hours per day [25]. In a third study electromagnetic fields (EMF) induced pronounced cortical bone formation [26]. EMF consisted of a sinusoid wave form with a maximum electromagnetic field of $10 \mathrm{G}$ and were applied for four hours per day. One study using osteoporotic rats did not demonstrate a beneficial effect on bone mass when treated with PEMF with $15 \mathrm{G}$ for 24 hours per day [27]. Furthermore, PEMF have also been shown to influence bone mass in disuse osteoporosis both clinically and experimentally [28-30].

Because treatment of osteoporosis with PEMF can have important consequences for today's standard treatment, we examined the effect of whole-body PEMF treatment on the bone microarchitecture in a rat model of osteoporosis. Cancellous and cortical bone changes in the proximal tibia were followed over time using in vivo microCT scanning.

\section{Methods}

Twenty female Wistar WU rats were obtained (Charles River, The Netherlands). All animals were housed in pairs in the institute's animal facility with a 12-hour light/dark regimen and received standard food pellets and water ad libitum. The study protocol was approved by the local Animal Experiments Committee (EUR 415) and was in accordance with Dutch law on animal experimentation.

At an age of 20 weeks a bilateral ovariectomy (OVX) was performed under sterile conditions to simulate osteoporosis. This was done under 3\% isoflurane gas anaesthesia (Rhodia Organique Fine Ltd., Bristol, UK). Buprenorphine $0.05 \mathrm{mg} / \mathrm{kg}$ per 12 hours (Schering-Plough, Kenilworth, NJ, USA) was given for pain relief for three days post-operatively.

The second day after OVX, PEMF treatment was started for five days/week over a period of six weeks. During treatment the animals were placed in a cage $(24 \times 30 \times 12 \mathrm{~cm})$ in pairs, with food and water ad libitum. The cage was surrounded by two connected coils normally used in the treatment of non-unions of the femur (IMD, Uden, The Netherlands) (Fig. 1). The rats were divided into five groups of four animals/group. The sample size was chosen for logistical reasons and not on the basis of a power analysis. The group sizes were kept relatively small because in vivo microCT analysis can accurately measure small changes over in time. A custom-made generator produced the

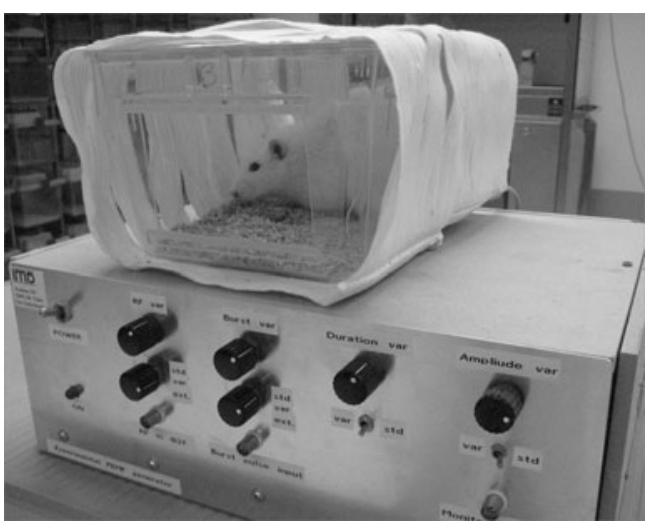

Fig. 1 Rat receiving systemic PEMF treatment. The cage is surrounded by coils. The custom-made generator can produce different electromagnetic signals

electromagnetic signals (see details below). The first group served as controls and was placed in a cage covered with non-functioning coils for two hours per day.

The second group was treated with a commercially available PEMF device that is effective for the treatment of nonunions and is clinically used (Orthopulse ${ }^{\circledR}$, IMD, Uden, The Netherlands) [31]. The device produces PEMF with a 1-G electromagnetic field, consisting of 5-ms pulse bursts with 5- $\mu \mathrm{s}$ pulses, and the bursts repeat at $15 \mathrm{~Hz}$. Rats were treated for two hours per day.

The treatment protocols of the third and fourth groups were designed to investigate whether a non-continuous PEMF stimulus would be more effective than a continuous stimulus, similar to studies investigating the effects of mechanical vibration on bone mass [32-34]. The third group received the same PEMF signal as the second group, but with a five minute on/off regimen; these animals were placed in the coil-covered cage for four hours per day to ensure that the total amount of PEMF time was the same in each animal. The fourth group also received the basic PEMF signal, but a custom-made amplifier added random noise of $50-150 \mathrm{kHz}$ over the basic stimulus. Animals were treated with this latter signal for two hours per day.

A fifth group received a PEMF signal that was not characterised by pulse bursts as in the other treatment groups, but consisted of quasi-rectangular single pulses of $1 \mathrm{G}$ given at $7.5 \mathrm{~Hz}$ with a pulse duration of $0.3 \mathrm{~ms}$. These animals were treated for two hours per day.

In vivo microCT scans were made at zero, three and six weeks after OVX. Using isoflurane (3\%) rats were anaesthetised and the right hind leg was scanned (voltage of $60 \mathrm{kV}$, current of $167 \mu \mathrm{A}, 0.5-\mathrm{mm}$ aluminium filter, $196^{\circ}$ with a rotation step of $1^{\circ}$ ) (SkyScan 1076 Microtomograph, Kontich, Belgium). Then 3D reconstructions with an isotropic voxel size of $18 \mu \mathrm{m}$ were made of the proximal tibia and the middiaphysis (Nrecon software version 1.5, SkyScan) (Fig. 2). The proximal metaphysis mainly consists of cancellous bone. 


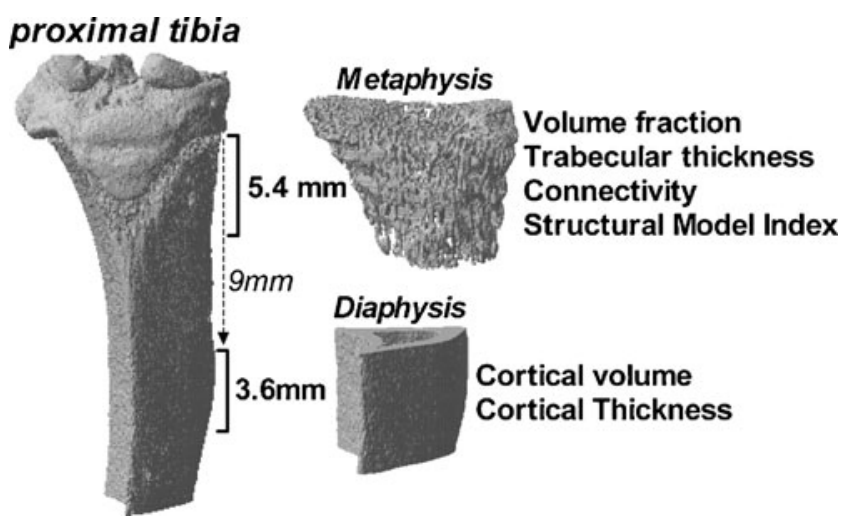

Fig. 2 Three-dimensional reconstruction of a microCT scan of the proximal tibia. The regions of interest in the metaphysis and diaphysis are indicated

A length of $5.4 \mathrm{~mm}$ was selected of which the epiphysis was manually deselected. The mid-diaphysis consists of cortical bone and the region of interest was selected $9 \mathrm{~mm}$ distal from the epiphysis and continued to $3.6 \mathrm{~mm}$ more distally. Using a local threshold, bone was separated from nonosseous structures, which resulted in binary data sets [35]. After automatic separation between trabecular and cortical bone using in-house software bone parameters were determined (3Dcalculator, SkyScan) [36]. On the metaphyseal region of interest, volume fraction (BV/TV), connectivity density (Conn/ TV), structure model index (SMI), in which an index of 3 indicates the presence of rods and an index of 0 indicates the presence of plates, and 3D trabecular thickness (TbTh) were determined. On the diaphyseal region of interest, cortical volume $(\mathrm{CtV})$ and cortical thickness $(\mathrm{CtTh})$ were determined.

Differences between means of the different treatment groups were statistically analysed using one-way analysis of variance (ANOVA) with a Tukey's multiple comparison test for each time point separately (GraphPad Software, San Diego, CA, USA).

\section{Results}

All surgical procedures were performed without complications. At the start of the study the average body weight of the rats was $224.6 \mathrm{~g}$ (SD 5.3). No period of weight loss was observed during the experimental period. The average weight gain was $57.6 \mathrm{~g}$ (SD 8.7) and there was no difference in weight gain between controls and any of the PEMF-treated groups.

In non-treated control animals BV/TV decreased from $31.7 \%$ (SD 4.8 ) at week zero to $24.2 \%$ (SD 4.1 ) and $19.7 \%$ (SD 3.5) at weeks three and six, respectively, as a consequence of OVX (Table 1). Morphometric parameters changed concomitantly, especially between weeks zero and three, with a decrease in mean TbTh, a decrease in Conn/TV and a structural change of trabeculae towards more rod-like
Table 1 Bone changes in the proximal tibia of ovariectomised nontreated control animals. Mean values with standard deviations are given $(n=4)$

\begin{tabular}{llll}
\hline & Week 0 & Week 3 & Week 6 \\
\hline $\begin{array}{l}\text { Volume fraction (\%) } \\
\begin{array}{l}\text { Trabecular } \\
\text { thickness }(\mu \mathrm{m})\end{array}\end{array}$ & $\begin{array}{l}\text { 127.7 (4.8) } \\
\text { Connectivity } \\
\text { density }\left(/ \mathrm{mm}^{3}\right)\end{array}$ & $\begin{array}{l}24.2(4.1) \\
125.0(3.7)\end{array}$ & $19.7(3.5)$ \\
$\begin{array}{l}\text { Structural model index } \\
\text { Cortical volume }\left(\mathrm{mm}^{3}\right)\end{array}$ & $1.7(0.19)$ & $2.1(0.15)$ & $2.2(0.09)$ \\
Cortical thickness $(\mu \mathrm{m})$ & $608.2(9.4)$ & $625.6(4.0)$ & $646.4(15.4)$ \\
\hline
\end{tabular}

structures, as apparent from an increase in SMI (Table 1). CtV increased from 12.3 (SD 0.23) $\mathrm{mm}^{3}$ at week zero to 12.9 (SD $0.23) \mathrm{mm}^{3}$ and 13.3 (SD 0.43) $\mathrm{mm}^{3}$ at weeks three and six, respectively. Mean $\mathrm{CtTh}$ also increased during this period.

None of the PEMF-treated groups showed a significant difference in $\mathrm{BV} / \mathrm{TV}$ or $\mathrm{CtV}$ compared with the control group at any time point (Fig. 3). The morphometric parameters also showed no significant differences compared with control animals. There were no indications to speculate the presence of any non-significant trend in the follow-up outcome variables.

\section{Discussion}

In this study we examined the effects of PEMF on bone changes in the cancellous and cortical bone during a six-week follow-up period. Although we used in vivo microCT scanning, which is a very sensitive analysis method, we were unable to reproduce the strong beneficial effects of PEMF on osteoporosis in ovariectomised rats as shown by others [12, 25, 26].

In this study four treatment protocols were used. Three of these protocols were based on a commercially available PEMF generator used for the treatment of non-unions. The fourth signal was developed as a single burst in order to be more comparable with the study of Chang and Chang [12]. In the study of Chang and Chang it was shown that PEMF prevented the trabecular bone loss induced by ovariectomy. Although one of the groups received PEMF with characteristics based on their signal, some differences in the experimental set-up still remain. Our signal had an electromagnetic current of $1 \mathrm{G}$ instead of $4-8 \mathrm{G}$ in the signal of Chang and Chang. Furthermore, they treated the rats for eight hours per day instead of two hours as used in our study. To examine whether longer exposure to PEMF would prevent further bone loss in our experimental set-up, we extended the experiment for an additional six weeks and treated the same animals for eight hours per day. However, trabecular bone loss was comparable to non-treated controls and no preventive effects of longer treatment could be found. 

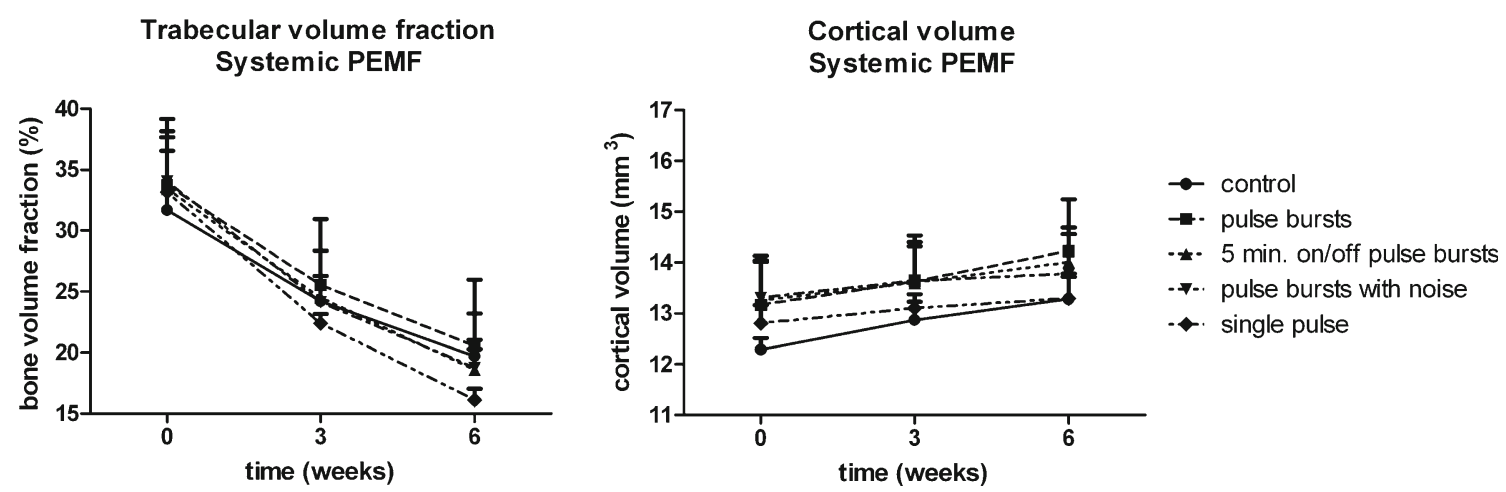

Fig. 3 Trabecular volume fraction and cortical volume in the tibia of ovariectomised rats that received PEMF treatment $(n=4)$ or received no treatment (controls, $n=4$ ). Data are mean values and standard deviations

In a more recent article PEMF, consisting of pulse bursts with an electromagnetic current of $9.6 \mathrm{G}$, were applied to ovariectomised rats during day time or night time [25]. In both groups PEMF treatment resulted in an increase in BMD and increased trabecular bone mass compared to ovariectomised controls. It was shown that treatment during daytime was more efficient than an overnight treatment. No effect on cortical bone was found. In another study in which ovariectomised rats were exposed to a $50-\mathrm{Hz}$ sinusoidal waveform of $10 \mathrm{G}$ an increase in cortical volume of $71 \%$ compared to non-treated controls was found [26]. One study has been published in which a negative finding is described. In that study 24 hours per day of systemic PEMF with a maximum magnetic field of $15 \mathrm{G}$ did not result in an increased bone mineral content after eight months of treatment [27].

Apart from differences in the characteristics of PEMF treatment used in each study, the ages of the animals also vary. In the studies that did find a positive effect of PEMF, eight to 13week-old rats were used $[12,25,26]$. In our study 20 -week-old rats were used and in the study of Takayama et al. five-monthold rats were used [27]. Rats younger than 20 weeks of age undergo extensive longitudinal growth, which probably affects the outcomes. For osteoporotic research it might be preferable to use skeletally mature animals, so that the results are easier to interpret in relation to the clinical situation.

Use of in vivo microCT scanning allows one to detect very small changes, because longitudinal data are collected. In the control group we observed an absolute decline in BV/TV of $7.5 \%$ (SD 1.63) at three weeks (Table 1). At a power of 0.8 , an $\alpha$ of 0.05 and with four animals per group, this gives a detectable alternative of $2.5 \%$ when comparing means of two groups. Thus, we would have been able to detect an effect of PEMF if the treatment had decreased trabecular bone loss to $5 \%$ or less compared to controls. Similar calculations for changes in $\mathrm{CtV}$ show that we would have been able to detect an effect of PEMF when cortical thickening was prevented by $0.25 \mathrm{~mm}^{3}$ or less given that control animals gain 0.6 (SD 0.17) $\mathrm{mm}^{3}$ of cortical bone in three weeks. In our opinion this is sufficient power because effects smaller than this would most likely not be of clinical significance. With this power we were also able to detect differences when bone changes at the magnitude of other studies were found.

In a clinical trial it was shown that by treating the proximal forearm of osteoporosis-prone women, an increase in BMD was induced at the distal radius of the treated side and also at the contralateral side, which suggests PEMF induced a systemic effect [23]. It is peculiar that this research and the strong beneficial effects in earlier animal experiments have not led to a range of clinical trials in which the effect of this safe and non-invasive intervention on bone mass is further examined. For many years attempts have been made to perform a prospective randomised controlled trial to determine the effect of PEMF on non-unions, but due to a lack of sufficient numbers for adequate statistical power this has never been achieved $[17,20,37,38]$. Obtaining sufficient numbers would not be a problem in a clinical trial examining the effects of PEMF on bone mass in healthy or osteoporotic individuals. Because PEMF does not induce any side effects and the exact experimental set-up is important for the outcome, it would be more logical and favourable to perform future studies on the effect of PEMF on bone in humans and not in animals. With the use of non-invasive techniques such as dual-energy X-ray absorptiometry (DXA) scanning and peripheral microCT scanning the role of PEMF in osteoporosis could be determined with certainty.

In this study we could not demonstrate a positive effect of PEMF on the bone architecture in a ovariectomised rat model of osteoporosis. This is in accordance with earlier published work. However, because there are studies that did find strong effects, it might be that PEMF are very effective under the right circumstances. It might therefore be more practicable to examine the effects of PEMF on bone mass directly in humans such that translation from small animal experiments is not an issue. However, the results obtained in our study do not substantiate a potential role for the use of PEMF in post-menopausal osteoporosis. 
Acknowledgments Funding was received from the Dutch Technology Foundation STW (RPG 6294).

Conflict of interest The authors declare that they have no conflict of interest.

Open Access This article is distributed under the terms of the Creative Commons Attribution Noncommercial License which permits any noncommercial use, distribution, and reproduction in any medium, provided the original author(s) and source are credited.

\section{References}

1. Watts NB, Bilezikian JP, Camacho PM, Greenspan SL, Harris ST, Hodgson SF, Kleerekoper M, Luckey MM, McClung MR, Pollack RP, Petak SM (2010) American Association of Clinical Endocrinologists Medical Guidelines for Clinical Practice for the diagnosis and treatment of postmenopausal osteoporosis. Endocr Pract 16 (Suppl 3):1-37

2. Rizzoli R, Reginster JY, Boonen S, Bréart G, Diez-Perez A, Felsenberg D, Kaufman JM, Kanis JA, Cooper C (2011) Adverse reactions and drug-drug interactions in the management of women with postmenopausal osteoporosis. Calcif Tissue Int 89(2):91104

3. Friedenberg ZB, Brighton CT (1966) Bioelectric potentials in bone. J Bone Joint Surg Am 48(5):915-923

4. Fukada E, Yasuda I (1957) On the piezoelectric effect of bone. J Phys Soc 12:1158-1162

5. Aaron RK, Wang S, Ciombor DM (2002) Upregulation of basal TGFbeta1 levels by EMF coincident with chondrogenesis-implications for skeletal repair and tissue engineering. J Orthop Res 20 (2):233-240

6. Bodamyali T, Bhatt B, Hughes FJ, Winrow VR, Kanczler JM, Simon B, Abbott J, Blake DR, Stevens CR (1998) Pulsed electromagnetic fields simultaneously induce osteogenesis and upregulate transcription of bone morphogenetic proteins 2 and 4 in rat osteoblasts in vitro. Biochem Biophys Res Commun 250(2):458-461

7. Fitzsimmons RJ, Ryaby JT, Mohan S, Magee FP, Baylink DJ (1995) Combined magnetic fields increase insulin-like growth factor-II in TE-85 human osteosarcoma bone cell cultures. Endocrinology 136(7):3100-3106

8. Guerkov HH, Lohmann CH, Liu Y, Dean DD, Simon BJ, Heckman JD, Schwartz Z, Boyan BD (2001) Pulsed electromagnetic fields increase growth factor release by nonunion cells. Clin Orthop Relat Res 384:265-279

9. Jansen JH, van der Jagt OP, Punt BJ, Verhaar JA, van Leeuwen JP, Weinans H, Jahr H (2010) Stimulation of osteogenic differentiation in human osteoprogenitor cells by pulsed electromagnetic fields: an in vitro study. BMC Musculoskelet Disord 11:188

10. Schwartz Z, Simon BJ, Duran MA, Barabino G, Chaudhri R, Boyan BD (2008) Pulsed electromagnetic fields enhance BMP-2 dependent osteoblastic differentiation of human mesenchymal stem cells. J Orthop Res 26(9):1250-1255

11. Hinsenkamp M, Collard JF (2011) Bone morphogenic proteinmRNA upregulation after exposure to low frequency electric field. Int Orthop 35(10): 1577-1581

12. Chang K, Chang WH (2003) Pulsed electromagnetic fields prevent osteoporosis in an ovariectomized female rat model: a prostaglandin E2-associated process. Bioelectromagnetics 24 (3):189-198
13. Nie K, Henderson A (2003) MAP kinase activation in cells exposed to a $60 \mathrm{~Hz}$ electromagnetic field. J Cell Biochem 90 (6):1197-1206

14. Schnoke M, Midura RJ (2007) Pulsed electromagnetic fields rapidly modulate intracellular signaling events in osteoblastic cells: comparison to parathyroid hormone and insulin. J Orthop Res 25(7):933-940

15. Adams BD, Frykman GK, Taleisnik J (1992) Treatment of scaphoid nonunion with casting and pulsed electromagnetic fields: a study continuation. J Hand Surg Am 17(5):910-914

16. Gossling HR, Bernstein RA, Abbott J (1992) Treatment of ununited tibial fractures: a comparison of surgery and pulsed electromagnetic fields (PEMF). Orthopedics 15(6):711-719

17. Griffin XL, Warner F, Costa M (2008) The role of electromagnetic stimulation in the management of established nonunion of long bone fractures: what is the evidence? Injury 39(4):419-429

18. Holmes GB Jr (1994) Treatment of delayed unions and nonunions of the proximal fifth metatarsal with pulsed electromagnetic fields. Foot Ankle Int 15(10):552-556

19. Madroñero A, Pitillas I, Manso FJ (1988) Pulsed electromagnetic field treatment failure in radius non-united fracture healing. J Biomed Eng 10(5):463-466

20. Mollon B, da Silva V, Busse JW, Einhorn TA, Bhandari M (2008) Electrical stimulation for long-bone fracture-healing: a metaanalysis of randomized controlled trials. J Bone Joint Surg Am 90(11):2322-2330

21. Hinsenkamp M, Ryaby J, Burny F (1985) Treatment of non-union by pulsing electromagnetic field: European multicenter study of 308 cases. Reconstr Surg Traumatol 19:147-151

22. Hinsenkamp MG (1982) Treatment of non-unions by electromagnetic stimulation. Acta Orthop Scand Suppl 196:63-79

23. Tabrah F, Hoffmeier M, Gilbert F Jr, Batkin S, Bassett CA (1990) Bone density changes in osteoporosis-prone women exposed to pulsed electromagnetic fields (PEMFs). J Bone Miner Res 5(5):437-442

24. Tabrah FL, Ross P, Hoffmeier M, Gilbert F Jr (1998) Clinical report on long-term bone density after short-term EMF application. Bioelectromagnetics 19(2):75-78

25. Jing D, Shen G, Huang J, Xie K, Cai J, Xu Q, Wu X, Luo E (2010) Circadian rhythm affects the preventive role of pulsed electromagnetic fields on ovariectomy-induced osteoporosis in rats. Bone 46 (2):487-495

26. Sert C, Mustafa D, Düz MZ, Akșen F, Kaya A (2002) The preventive effect on bone loss of 50-Hz, 1-mT electromagnetic field in ovariectomized rats. J Bone Miner Metab 20(6):345-349

27. Takayama K, Nomura H, Tanaka J, Zborowski M, Harasaki H, Jacobs GB, Malchesky PS, Licata AA, Nosé Y (1990) Effect of a pulsing electromagnetic field on metabolically derived osteoporosis in rats: a pilot study. ASAIO Trans 36(3):M426-M428

28. Eyres KS, Saleh M, Kanis JA (1996) Effect of pulsed electromagnetic fields on bone formation and bone loss during limb lengthening. Bone 18(6):505-509

29. Rubin CT, Donahue HJ, Rubin JE, McLeod KJ (1993) Optimization of electric field parameters for the control of bone remodeling: exploitation of an indigenous mechanism for the prevention of osteopenia. J Bone Miner Res 8(Suppl 2):S573-S581

30. Skerry TM, Pead MJ, Lanyon LE (1991) Modulation of bone loss during disuse by pulsed electromagnetic fields. J Orthop Res 9 (4):600-608

31. Punt BJ, den Hoed PT, Fontijne WPJ (2007) Pulsed electromagnetic fields in the treatment of nonunion. Eur J Orthop Surg Traumatol 18:127-133

32. Castillo AB, Alam I, Tanaka SM, Levenda J, Li J, Warden SJ, Turner CH (2006) Low-amplitude, broad-frequency vibration effects on cortical bone formation in mice. Bone 39(5):1087-1096

33. Saxon LK, Robling AG, Alam I, Turner CH (2005) Mechanosensitivity of the rat skeleton decreases after a long period 
of loading, but is improved with time off. Bone 36(3):454464

34. Tanaka SM, Alam IM, Turner CH (2003) Stochastic resonance in osteogenic response to mechanical loading. FASEB J 17(2):313314

35. Waarsing JH, Day JS, Weinans H (2004) An improved segmentation method for in vivo microCT imaging. J Bone Miner Res 19 (10):1640-1650
36. Erasmus M (2011) The Erasmus Orthopaedic Research Laboratory vol. Rotterdam

37. Bray TJ (1995) A prospective, double-blind trial of electrical capacitive coupling in the treatment of non-union of long bones. J Bone Joint Surg Am 77(5):809

38. Barker AT, Dixon RA, Sharrard WJ, Sutcliffe ML (1984) Pulsed magnetic field therapy for tibial non-union. Interim results of a double-blind trial. Lancet 1(8384):994-996 